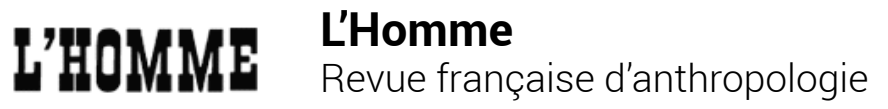 \\ 165 | janvier-mars 2003 \\ Image et anthropologie
}

\section{Pouvoir ou efficacité symbolique des images}

\section{Bertrand Prévost}

\section{(2) OpenEdition \\ Journals}

\section{Édition électronique}

URL : http://journals.openedition.org//homme/15602

DOI : 10.4000/lhomme.15602

ISSN : 1953-8103

\section{Éditeur}

Éditions de l'EHESS

\section{Édition imprimée}

Date de publication : 1 janvier 2003

Pagination : 275-282

ISBN : 2-7132-1779-2

ISSN : 0439-4216

\section{Référence électronique}

Bertrand Prévost, «Pouvoir ou efficacité symbolique des images », L'Homme [En ligne], 165 | janviermars 2003, mis en ligne le 27 mars 2008, consulté le 24 septembre 2020. URL : http://

journals.openedition.org//homme/15602 ; DOI : https://doi.org/10.4000//homme.15602 


\title{
Pouvoir ou efficacité symbolique des images
}

\author{
Bertrand Prévost
}

L'

OUVRage de David Freedberg, Le Pouvoir des images, s'impose comme un "classique" dans le champ de l'histoire de l'art". Sa traduction récente en français est donc particulièrement bienvenue.

Le pouvoir des images. D'emblée le titre annonce qu'il ne sera pas tant question d'art que d'image. Dès l'introduction, l'auteur s'attache à " prendre délibérément en compte toutes les images, et non pas seulement celles que l'on considère comme artistiques, [en] aboli[ssant] cette distinction ancienne entre les objets qui suscitent certaines réactions en raison de leur pouvoir "magique" ou "religieux" présumé, et ceux qui sont censés remplir des fonctions purement "esthétiques" " (pp. 9 et 11). Le point de départ théorique de l'auteur consiste à se défaire radicalement de l'esthétisme d'une histoire de l' a rtdominante informée par un kantisme et/ou un hégélianisme qui, le plus souvent, s'ignorent. "Le pouvoir des images s'oppose au traditionnel point de vue kantien sur l'art» (p. 6). Premier principe : l'artn'est en rien une activité désintéressée; l'art ne vise pas un Beau éternel et idéal, ni un plaisir pur. C'est à ce prix qu'une pensée en termes d'image et non d'a rt se révèle bien plus opératoire pour appréhender le difficile problème de l'efficacité iconique. Il aura d'ailleurs fallu les travaux du grand historien de l'art, Hans Belting, pour se faire à l'idée que la posture de l'esthète n'a en soi rien d'évident, que la " réaction distinguée $»^{2}$ possède déjà toute une histoire qui ne saurait être réduite à une histoire de l'art mais plutôt au passage d'une "époque de l'image " à " une époque de l'art",

1. Ce livre a suscité de vives réactions dans la discipline, sous la forme d'incompréhensions et de débats souvent stériles. Cf. par exemple John M. Nash, "Art and arousal », Art Histoy» 1990, XIII (4) : 566-570, et la réponse de l'auteur dans Art History 1992, XV (2) : 275-278.

2. L'auteur dit vouloir « conjurer le spectre de la réaction distinguée (the phantom of high response)» (p. 17).

À propos de David Freedberg, Le Pouvoir des images. Trad. de l'américain par Alix Girod. Paris, Gérard Monfort, 1998. [Éd. orig.: The Power of Images. Studies in the History and Theory of Response, Chicago \& London, The University of Chicago Press, 1989.] 
d'un « a rt du culte » à " une culture de l' a rt », transformation aussi lente que relevant d'une temporalité complexe ${ }^{3}$.

$\mathrm{Ce}$ "pouvoir » de l'image ne pourra d'abord se repérer que dans ses effets, que dans la réaction (response) des hommes face aux images. Autrement dit, cela implique de (ré)insérer l'image dans un champ de pratiques afférentes : à l'opposé de l'esthétisme dominant de l'histoire de l'art, l'auteur prend le parti d'un pragmatisme visant l'image non pas tant comme représentation du monde que comme action sur le monde. C'est ce que Freedberg nomme - un peu rapidement - dans son chapitre conclusif la "réalité de l'image » : "il convient désormais d'envisager la peinture et la sculpture dans une plus grande continuité avec la réalité que nous avons été accoutumés à qualifier de telle, et de réinsérer la figuration et l'imitation dans la réalité (ou, plus exactement, dans notre expérience de la réalité); $[\ldots]$ accorder à toutes les images leur pleine valeur de réalité et non seulement (pour simplifier, à l'ancienne mode) de représentation " (p. 471). L'image sera donc (enfin) regardée avec, si l'on peut dire, son opacité objectale, son poids d'objet lesté d'expérience, chargé de pratiques rituelles ou non: une image saisie dans son épaisseur anthropologique ${ }^{4}$.

Les chapitres de l'ouvrage brassent ainsi un très riche matériel et font de celui-ci une sorte de somme tout aussi remarquable qu'indispensable sur les pratiques de l'image. Il ne sera pas question ici de discuter ce matériel en détail, mais de le considérer dans les présupposés, les implications et les conséquences théoriques qui en orientent l'exposition, puisque l'auteur a avant tout voulu défendre une thèse. On remarquera tout de suite la place privilégiée qu'occupent les images religieuses: le livre ne cesse de rencontrer le problème de l'idole (ou plutôt de l'idolâtrie) en s'ouvrant sur la question des images de culte (chap. II), considérée plus loin sous l'angle du rituel de présentification (chap. V), du pèlerinage (chap. VI), des récits de visions (chap. XII). Il importe de bien comprendre qu'une image de culte n'est pas tant faite pour être vue que pour voir, et que l'« échange » de regards entre elle et le dévot spectateur demeure foncièrement dissymétrique : l'image regardera avant même d'être vue, son spectacle, sa visibilité relèveront plus du don (l'image se donnant à voir) ou de la «capture» du regard (l'image fascinante) que de l'échange. Particulièrement exemplaires sont donc les rituels qui font de l'ouvertu re des yeux l'acmé de la consécration d'une image. C'est le cas de la "cérémonie des yeux » des bouddhistes cinghalais du Theravada, au cours de laquelle les yeux de la statue de Bouddha, qui seuls lui manquent, sont enfin peints : «La cérémonie est considérée par les exécutants comme périlleuse et est entourée de tabous. Elle est accomplie par l' a ruisan qui a fabriqué la statue, au terme d'une cérémonie de plusieurs heures pour s'assurer que nul mal ne lui adviendra. [...] L'artisan peint les yeux à un moment propice et

3. Cf. Hans Belting, Bild und Kult. Eine Geschichte des Bildes vor dem Zeitalter der Kunst, Munich, Beck, 1990. La récente traduction française de ce grand livre (Image et culte. Une histoire de l'art avant l'époque de l'art, Paris, Cerf, 1998) gomme toute la subtilité du sous-titre allemand qui jouait sur l'opposition image/art.

4. On pensera à la notion d’ "image-objet " développée par Jérôme Baschet dans L'Image. Fonctions et usages des images dans l'Occident médiéval, Paris, Le Léopard d'Or, 1996 : 7-26 ("Cahiers du Léopard d'Or " 5). 
demeure seul dans le temple fermé avec ses seuls aides, tandis que chacun, à l'extérieur, se tient à distance de la porte. En outre, l'a artisan ne se risque pas à regarder la statue en face, mais il s'en détourne et peint de côté ou par-dessus son épaule, en regardant dans un miroir qui capte le regard de l'image à laquelle est conférée la vie. À peine la peinture achevée, le regard de l'artiste lui-même devient dangereux. L'homme est alors emmené au-dehors les yeux bandés et le bandeau n'est retiré que lorsque son regard pourra tomber sur un objet qu'il détruit alors symboliquement d'un coupd'épée» (p. 105).

Lauteur passe également en revue les pratiques votives, mixtes de sacré et de profane (chap. VII, qu'il faut lire conjointement avec le chap. IX sur le portrait votif en cire), et ces pratiques d'envoûtement par l'image mettant en ouvre une damnatio memoriae (chap. X). C'est ici que Freedberg évoque les «images infamantes» dont l'Italie médiévale et renaissante s'est fait une spécialité. Loin d'être marginale, l'utilisation d'images d'infamie était des plus officielles et s'ancrait dans des contextes judiciaire, civique ou politique. Il s'agissait de châtier un criminel, un adversaire politique ou tout autre ennemi public en exposant son image, en l'injuriant, en la défigurant, voire en la détruisant. Cela amène enfin l'auteur à consacrer une place aux questions de l'aniconisme (chap. IV), de la censure (chap. XIII), de l'iconoclasme et du vandalisme à propos des images (chap. XIV) .

Au vu de ce seul dépouillement (encore que partiel) et de l'accumulation des phénomènes considérés, on pèsera l'hétérogénéité fondamentale qui caractérise les usages de l'image dans son devenir occidental. Dès son introduction, l'auteur avait revendiqué une certaine généralité: "Notre première démarche doit être celle de l'ethnologue et consiste à rassembler le maximum de matériel sur toutes les catégories de la société; il faut ensuite se ranger à l'anthropologie culturelle et envisager un éventail de sociétés aussi étendu que possible» (p. 37). L'auteur n’hésite donc pas à faire des incursions dans l'Égypte, la Mésopotamie et la Grèce anciennes ainsi que dans les sociétés dites primitives. Lépistémologie de Freedberg procède selon les modèles du type, de la catégorie et de l'exemple : «Notre démarche est bel et bien inductive. Tout en m’attachant à l'étude minutieuse d'éléments isolés [...], j'envisage l'ensemble des relations humaines avec les images afin de mettre en évidence des aspects du comportement et la réaction (response) dont on puisse reconnaître le caractère universel et la permanence d'une culture à une autre » (p. 38) ${ }^{5}$. C'est donc par l'ampleur et la quantité de faits particuliers que l'on atteindrait un niveau d'universalité ; il y aurait comme un pont entre la généralité du discours et la prétention à fonder des principes a priori. Freedberg tente de lier synthétiquement la diversité de son matériel empirique dans l'unité d'un grand principe transcendantal.

5. Cf. aussi p. 10 : «J'espérais tro u ver un registre de discours plus général [...] »; «Je décidai que la meilleure façon de procéder serait d'exposer certains aspects de réactions récurrentes [...] ; "Dans l'exposition des témoignages, il était clair que les distinctions fondamentales devaient s'opérer par catégories de réactions plutôt que par catégories d'images »; et p. 469 : « une histoire de la réaction ne peut s'entreprendre qu'en prenant en considération la plus grande variété possible d'images ", etc. (mes italiques, B. P.). 
Le motif récurrent de l’ouvrage, le mot clé quasi «magique» de Freedberg, le principe le plus général qui informe nos réactions face aux images, l'auteur l'appelle refoulement (repression): "L'être humain est stimulé sexuellement par les peintures et les sculptures; il les brise, les mutile, les embrasse, pleure devant elles, voyage pour les rencontrer; face à elles, il est apaisé, ému, poussé à la révolte. Par elles, il exprime sa gratitude, espère s'élever, éprouve au suprême degré empathie et crainte. C'est ainsi qu'il a toujours réagi, qu'il réagit encore, dans les sociétés dites primitives et dans les sociétés modernes, en Orient et en Occident, en Afrique, en Amérique, en Eu rope et en Asie. [...] Ce qui m’intéresse, ce sont les réactions (responses) vouées au refoulement (repression), pa ree que trop embarrassantes, vulgaires, grossières, incultes, parce qu'elles nous font prendre conscience de notre lien avec l'ignorance, la grossièreté, le primitif, le latent; et parce qu'elles ont des racines que nous jugeons inavouables» (p. 21). Voilà donc la souche et ses racines psychologiques qui seraient le fondement de la question du "pouvoir» des images. Sans même préjuger de son assise, un vent d'inquiétude soufflera quant à sa simple rigueur, puisque le même auteur, quelques pages plus haut (p. 10), avouait que « [sa] philosophie [était] simple et [sa] psychologie élémentaire »...

Or, c'est ce souci de généralité qui l'empêche de penser véritablement l'efficacité iconique, le problème n'étant en fait jamais abordé, toujours présupposé, donc impensé $^{6}$. Si Freedberg a parfaitement compris l'intérêt épistémologique qu'il y a à parler en termes d'images plutôt que d’art, à l'autre pôle de sa question il reste dans une visée totalisante en envisageant le problème sous l'angle " $d u$ pouvoir ", en considérant celui-ci comme une entité idéale, comme un universel : le pouvair des images, éternel, unique et identique à lui-même, depuis l'Égypte antique jusqu’à l'art moderne et contemporain. Cela n'implique pas du tout qu'il faille en rester à un niveau empirique et que des phénomènes de longue durée, structuraux pour le dire vite, soient des chimères, bien au contraire. Tout travail sérieux, je veux dire tout travail qui ne se repait pas de l'anecdotique, doit pouvoir dessiner un horizon "méta-empirique », mais où toute la difficulté épistémologique tient dans le risque que celui-ci ne cache en réalité un au-delà métaphysique (précisément celui qui informe les ouvrages historiques dits de "grandes synthèses»). En dautres termes, c'est le faux problème de l'opposition de l'universel et du particulier qu'il faut savoir dépasser. Une «structure », pour nommer cela trop rapidement, ne se construit pas sur une somme d’exemples à valeur généralisante; elle se repère dans des singularités, c'est-à-dire dans des phénomènes, aussi microscopiques soient-ils, qui sont porteurs de complexité, qui s'imposent par la différence qu'ils mettent en œuvre. Il

6. C'est d'ailleurs ce que faisait déjà remarquer Louis Marin quant aux difficultés - voire à l'aporie - qu'il y a à appréhender le " pouvoir » des images du seul point de vue de la catégorie générale des effets, d'une généralisation des effets iconiques (cela dit en faisant explicitement référence à l'ouvrage de Freedberg); «la force de l'image, en son fond, c'est-à-diredans son origine, sa fonction, son but et sa fin, ne sera jamais qu' induite et présupposée dans les configurations spécifiques qu'elle pourra prendre dans l'histoire et la culture, à partir de ses effets et de leur raison, pour employer l'expression pascalienne, toute formulation ontologique concernant son essence - l'être de la force - n'étant qu'une fiction théorique relevant d'une hypothèse métapsychologique (ce qui ne veut pas dire qu'elle n'ait pas lieu dêtre introduite [ou induite] et travaillée) ", in Louis Marin, Des pouvoirs de l'image. Gloses, Paris, Seuil, 1993: 15. 
y aurait là un grand avantage à tirer parti de la notion de multiplicité au sens où Gilles Deleuze parlait d'une multiplicité : bâtir non pas des ensembles numériques d'images, où les relations entre elles seraient conçues dans des rapports de cause à effet ou d'influence, mais des amas ouve rts aux contours flous, liés par des intensités, des nœuds dont on ne sait jusqu'où les fils entrelacés nous emmèneront, tout ce que Deleuze- avec Bergson - appelait «multiplicité distincte ", " qualitative», ou encore « virtuelle».

Du reste, cet échec méthodologique est d'autant plus étonnant que l'auteur a placé son ouvrage sous l'égide d'un épigramme de Guichardin exhortant à cette pensée des singularités ${ }^{7}$. Or, cette citation, un autre historien de l'art en avait déjà fait usage et avait su, quant à lui, en tirer tout le bénéfice méthodologique - il s'agit d'Aby Warburg ${ }^{8}$. C'est en partant d'un cas, d'une singularité (les portraits de Francesco Sassetti dans sa chapelle à Santa Trinità de Florence) ouverte à une multiplicité de temps et de pratiques que Warburg avait pu mettre à jour l'efficacité à l'œuvre dans les portraits de la première Renaissance florentine'. En effet, l'historien de l'art avait rendu impensable l'étude du portrait dans la Florence de la Renaissance sans celle de ces effigies en cire, ces portraits hyperréalistes puisque moulés sur le visage et montés sur un mannequin, que les riches Florentins venaient déposer en ex-voto dans l'avant-cour de l'église de la Santissima Annunziata.

Parler « du " pouvoir des images, autrement dit chercher dans l'image le principe de son efficacité, cela revient en fait à hypostasier, à substantialiser ce dit pouvoir : à faire de l'image une chose. Or, à prop rement parler, l'image n' "a " pas de pouvoir, comme si celui-ci pouvait se posséder, être l'objet d'une propriété. En ne considérant l'image que comme le terme d'une relation, d'une "interaction " avec un spectateur, Freedberg oublie que l'image elle-même est une relation. Son efficacité suppose toujours qu'elle soit le lieu ou la configuration d'un rapport de forces qui prenne et englobe déjà le spectateur. On posera mal le problème de l'efficacité iconique tant que l'on restera dépendant d'une opposition, d'une découpe sujet (spectateur) / objet (image) - opposition philosophiquement idéaliste, donc aporétique. Or, le projet de

7. « $\mathrm{E}$ grande errore parlare delle cose del mondo indistintamente e assolutamente, e, per dire così, per regola; perché quasi tutte hanno distinzione ed eccezione per la varietà delle circumstanze, in le quali non si possono fermare con una medisima misura : e queste distinzioni ed eccezioni non si trovano scritte in su'libri, ma bisogna lo insegni la discrezione" (Francesco Guicciardini, Ricordi politici e civili, a cura di Adolfo Faggi, Milano, Unione tipografica-editrice torinese, $1945: 20$ ). "C'est une grande erreur que de parler des choses du monde sans distinctions et dans l'absolu et, pour ainsi dire, selon une règle; parce qu'elles présentent presque toutes des distinctions et des exceptions dues à la diversité des circonstances, dont on ne peut rendre compte par une mesure unique : et ces distinctions et exceptions ne se trouvent pas écrites dans les livres, mais c'est au discernement de les enseigner " (ma traduction, B. P.).

8. Cf. Aby Warburg, "Bildniskunst und florentinisches Bürgentum» (1902), in Gert rud Bing, ed., Gesammelte Schriften, Leipzig, Berlin, Teubner, 1932, Bd. I : 89-126. [Traduit de l'allemand par Sibylle Muller sous le titre, "L'art du portrait et la bourgeoisie florentine ", in Essais florentins, Paris, Klincksieck, 1990 : 101-135 (la traduction française omet l'épigramme deGuichardin)].

9. Pour une étude de l' a rticle de Warburg et de ses conséquences épistémologiques, cf. Georges DidiHuberman, "Pour une anthropologie des singularités formelles. Rema rque sur l'invention warburgienne", Genèses, 1996, 24 : 145-163. Bien évidemment, Freedberg n’est pas sans connaître l'étude de Warburg, qu'il cite d'ailleurs à plusieurs reprises (p. 184, sur la question des ex-voto, et pp. 271-273, sur la question du portrait en cire), mais il ne s'en sert qu'au titre de matériau historique, manquant toute la dimension épistémologique. 
l'auteur était justement d'élaborer une histoire et une théorie de la response, de la "réaction ", autrement dit d'isoler en tant que pôles fixes un spectateur et une image et de repérer ses effets sur ce spectateur.

À cet égard, il semble que l'auteur se laisse prendre à ce "pouvoir ", qu'il en soit le jouet, qu'il en subisse les effets: un effet de croyance. Car toute l'efficacité de l'image consiste précisément en ce qu'elle instaure la croyance en un pouvoir qui lui serait inhérent, en une force qui serait indépendante de tout rapport de forces. Le tour de passe-passe de l'image est de s'emparer, de configurer des puissances, puis de donner l'illusion qu'elle les possède en pro pre, que son efficacité, son " pouvoir ", relève d'une propriété intrinsèque. L'efficacité del'image tient alors dans l'opération disolation qui vient défaire en les occultant les rapports de forces qui la traversent, qui vient couper l'image des relations concrètes dans lesquelles elle est prise. Or cela, c'est précisément ce que mettent en acte les rituels de consécration par lesquels une puissance (une divinité, par exemple) est présentifiée (rendue présente, et non représentée), par lesquels un simple m o reau de bois ou un chef-d' œuvre de la Renaissance deviennent des objets de culte, des images efficaces.

Il faut reconnaître à l'auteur d'avoir vu là quelque chose d'important : " étudier l'histoire des images, c'est étudier l'histoire de la consécration [...] : la consécration d'une image la fait fonctionner ou, pour le moins, apporte un changement dans la manière dont elle fonctionne» (p. 103). Mais affirmer cela, n'est-ce pas produire une tautologie, se soumettre à une injonction à croire? Puisque les rituels, les liturgies et les cérémonies effectués sur l'image incluent et présupposent toujours la croyance, puisqu'ils l'effectuent et la reproduisent bien plus qu'ils ne l'instaurent, la description de tels actes n'expliquera rien sur le fonctionnement efficace d'une image. Ce sera encore subir la croyance, être pris par elle. Or l'histoire ou l'anthropologie est bien ce qui doit penser la croyance et non être pensé par elle. D'ailleurs, Freedberg concède quelques pages plus loin que "la consécration, par elle-même, n'est rien. Elle constitue un but, une visée; elle fait face. Elle porte en elle ses conséquences. On ne peut la définir qu'en fonction de la manière dont elle rend les images efficaces. Ce n'est pas à dire que la consécration déclenche le pouvoir effectif d'une image. Celle-ci peut être opérante avant la consécration [...]. Les images agissent précisément parce qu'elles sont consacrées, mais également avant de l'être" (pp. 115-116 ; traduction modifiée par l'auteur, B. P.).

Cette dernière phrase me semble importante car elle souligne la nécessité d'une double approche de la question. D'abord, tenter de comprendre quelque chose à l'efficacité iconique suppose l'élaboration d'une phénoménologie. Une phénoménologie qui ne cherche pas à expliquer mais s'attache à décrire patiemment et précisément des images dans toute leur efficacité formelle: "Je ferai le choix de suggérer Pimportance primordiale de la relation entre qualité formelle et efficacité des objets" (p. 315). Il s'agit de se demander comment, dans une image (qu'elle soit matérielle ou mentale), une puissance se donne à voir, sous quelles transformations et déformations elle devient "présentable"; autrement dit, comment opère un processus que Freud avait nommé figurabilité 
(Darstellbarkeit) dans le cadre de l'image de rêve ${ }^{10}$. Dans notre contexte, un tel travail serait par exemple à étudier dans le face à face fascinant, quasi hypnotique de la Véronique. Comment ne pas songer ici à ce que Walter Benjamin nommait une aura, et dont il avait donné les prémisses pour une phénoménologie en en parlant comme d'une « unique apparition d'un lointain, si proche qu'elle puisse être " ${ }^{11}$ ? En d'autres termes, s'enquérir des formes (de figurabilité) qu'a pu revêtir le sacré dans notre civilisation.

En outre, cela n'a d'intérêt qu'à faire tenir ensemble l'a pp roche formelle et l'approche anthropologique, morphologie et histoire de la culture. Il ne s'agit pas de dire que ce sont deux niveaux d'analyse complémentaires, mais de les entendre dans un rapport de métamorphose. Il resterait à voir comment les usages et les fonctions d'une image se configurent dans et par l'image, y trouvent une consistance formelle sous un mode qu'on dira précisément de transformation. Une image ne se contente pas de représenter, de reproduire du réel et de l'expérience vécue, elle les transforme; ćest-à-dire qu'elle ouvre un lieu, à la fois réceptacle et producteur, qui vient informer différentiellement une partie du monde social, qui vient donner une chair d'image à des pratiques sociales. C'est à travers la singularité formelle des portraits de Sassetti, autant par leur style hyperréaliste que par l'étrangeté du dispositif formel de la chapelle Sassetti, que Warburg avait pu les mettre en rapport avec les boti en cire.

Mais cela ne saurait suffire: si, dans un premier temps, il s'agissait de rester en deçà de la croyance, de demeurer dans l'élément des représentations culturelles, il faut, dans un second temps, en venir à un autre questionnement; dépasser ce qui, sous le nom de phénoménologie, était une histoire naturelle (aussi subtile fût-elle, elle s'attachait aux contenus culturels des représentations) pour accéder au niveau prop rement anthropologique (si ce n'est philosophique) des conditions de l'efficacité d'une image ${ }^{12}$. C'est-à-dire, interroger l'activité représentative elle-même, la représentation dans son mouvement dynamique : qu'est-ce que cela fait - ou plutôt qu'est-ce qui fait - que l'on a pu des siècles durant rendre un culte aux images, les utiliser à des fins magiques, ou qu'aujourd'hui encore, on les collectionne et les vénère dans nos musées sous couvert de "dévotion esthétique "? Formuler ainsi

10. Sur cette féconde notion de l'esthétique contemporaine (retravallée par Lyotard, Deleuze ou DidiHuberman, pour ne citer qu’eux), je me contenterai de renvoyer au récent article, éclairant et faisant le point, d'Olivier Schefer, «Qu'est-ce que le figural ? », Critique, 1999, 630 : 912-925.

11. Walter Benjamin, "L'œuvre d' a rt à l'è re de sa re productibilité technique " (1936), traduit de l'allemand par Maurice de Gondillac, Essais, Paris, Denoël, 1971, 2: 94. Freedberg convoque dailleurs cette notion dès la page 6 de son ouvrage. Il faudrait reli rece très célèbre texte conjointement avec la phénoménologie du sacré de Rudolf Otto, Le Sacré. L'élément non rationnel dans l'idée du divin et sa relation avec le rationnel (1917), traduit de l'allemand par André Jundt, Paris, Payot, 1995. Pour une phénoménologie de l'aura, voir entre autres Georges Didi-Huberman, Ce que nous voyons, ce qui nous regarde, Paris, Minuit, $1992: 103-123$.

12. Sur ce double niveau d'une histoire naturelle et d'une phénoménologie, on peut lire les belles pages de Paul Veyne dans Le Pain et le cirque. Sociologie historique d'un pluralisme politique, Paris, Seuil, 1976 : 523-533 ("Appendice: Les dieux. Histoirenaturelle ou phénoménologie?»), qui donnent le programme théorique, dans le sens d'une anthropologie de la croyance religieuse, d'un autre livre du même auteur, Les Grecs ont-ils cru à leurs mythes? Essai sur limagination constituante, Paris, Seuil, 1983 (« Point Essais»). 
cette question incite à penser l'image dans les termes anthropologiques d'une efficacité symbolique - question encore largement ouverte dans le champ de l'anthropologie, à peine abordée dans celui de l'histoire de l'art. Cette question est cruciale car elle me semble être le fond de tout ce qui se rapporte à une anthropologie de l'image. Qu'est-ce qui fait que l'image fonctionne? Qu'est-ce qui fait que "ça marche"? Comment passe-t-on d'une représentation à une action? "How to do Things with Images?», pour le dire en pastichant le titre d'un célèbre ouvrage d'Austin. C'est précisément sur ce point quachoppe l'entreprise de Freedberg ${ }^{13}$ en focalisant son attention sur un prétendu " pouvoir » des images, sans oser ouvrir la question à la problématique plus vaste - et plus difficile - d'une efficacité anthropologique dont le noud ou l'horizon se situerait dans une anthropologie de la croyance, dans le rapport fondamental que la croyance entretient avec la culture. On sait que c'est parce que Marcel Mauss se serait laissé «mystifier " par une croyance indigène que Claude Lévi-Strauss a critiqué son "Essai sur le don ": Mauss pris au piège du mana, au fond incapable d'en rendre compte autrement qu'en reconduisant une explication quil se devait justement dexpliquer ${ }^{14}$. Sans parler des critiques que cette critique a pu susciter ${ }^{15}$, c'est toujours la question de la créance (au double sens de dette et de croyance) comme fondement du social qui refait surface quand on aborde l'épineux problème de l'efficacité symbolique.

MOTS CLÉS/KEYWORDS: image - pouvoir/pouer - efficacité symbolique/symbolic efficiency culte/cult - aoyancelbelief.

13. Ce point d'achoppement se remarque, notamment chez Freedberg, dans des remarques pour le moins fort naïves (et dans un style très "positiviste façon XIXe siècle"); ainsi, dans un chapitre intitulé «Images vivantes : valeur des visions et des récits » : "Il s'agit indéniablement et significativement de ps ydhologie populaire. [...] On alléguera qu'aucun individu doué de sens critique n’a sans doute jamais cru qu'une statue était vivante, et que toute confusion éventuelle n’a pu se manifester que dans les classes populaires" (pp. 318, 321). Dans ce genre de situation, quand un historien ne parvient pas à cerner un problème comme il faut, le "peuple " a toujours bon dos!

14. C f. bien entendu l'" Introduction à l' $œ u$ v re de Ma reel Mauss" (1950), in Ma rcel Mauss, Sociologie et anthropologie, Paris, PUF, 1995 ( $5^{\mathrm{e}}$ éd.) : IX-LII.

15. Par exemple et récemment, celles, très pertinentes, de Maurice Godelier dans L'Énigme du don (Paris, Fayard, 1996) qui focalisent l'attention sur ce qui, dans une société, ne s'échange précisément pas, à savoir les biens et les objets inaliénables. 